\title{
Pulmonary and mediastinal tuberculosis in an immunocompetent child presenting as sternal abscess protruding from the skin
}

\author{
B. Gülhan · H. Özdemir · S. Kanik-Yüksek $\cdot$ H. Tezer
}

Received: 18 September 2014 / Accepted: 15 November 2014 / Published online: 26 November 2014

(C) Springer-Verlag Berlin Heidelberg 2014

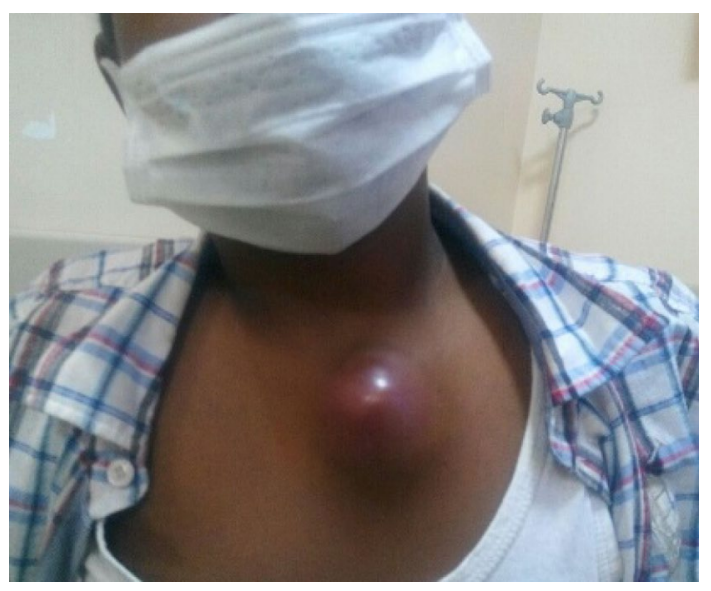

Fig. 1 Swelling abscess of the patient sized $4 \times 4 \mathrm{~cm}$

A 10.5-year-old otherwise healthy male patient, coming from Somalia, admitted to our centre because of a swelling lesion on his chest over the sternum. The abscess had slowly grown over a period of 2 months. He had not have cough, fever and weight loss. There was a $4 \times 4 \mathrm{~cm}$ sized fluctuating abscess on the skin over sternum in physical examination (Fig. 1). Bacille Calmette-Guérin (BCG) scar was positive on his left shoulder. Infection markers were negative. Contrast-enhanced computed tomography (CT) had showed subcarinal $43 \times 30 \mathrm{~mm}$ sized with central necrosis in the middle of lesion, multiple mediastinal and hilar lymphadenopathies, $60 \times 40 \mathrm{~mm}$ sized abscess close

B. Gülhan $(\varangle) \cdot$ H. Özdemir $\cdot$ S. Kanik-Yüksek $\cdot$ H. Tezer Ankara Children's Diseases Hematology Oncology Training Hospital, Ziraat Mah. İrfan Baştuğ Cad. Kurtdereli Sk. No:10 Dışkapı, Ankara 06110, Turkey

e-mail: docbelgin@yahoo.com

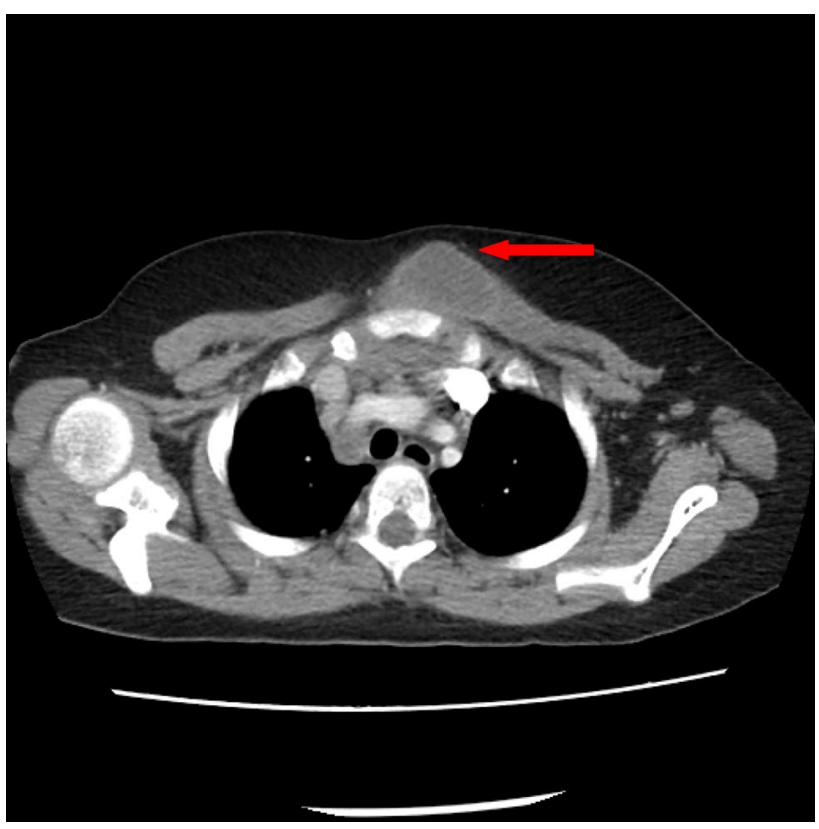

Fig. 2 Protruding abscess from sternum of the patient is seen with arrow

to arcus aorta that destructed of sternum and reached out to subcutaneous tissue and centrilobular nodules and tree-inbud sign in upper lobe of right lung. He was hospitalized and diagnosed with pulmonary and mediastinal lymph node tuberculosis. Spontaneous drainage of abscess occured. As an acid-fast bacilli, polymerase chain reaction (PCR) and culture for Mycobacterium tuberculosis complex were positive in abscess material, a diagnosis of pulmonary and mediastinal TB was made and oral anti-TB therapy was started. Subtype analysis of material was compatible with Mycobacterium tuberculosis. Immune deficiency and HIV 


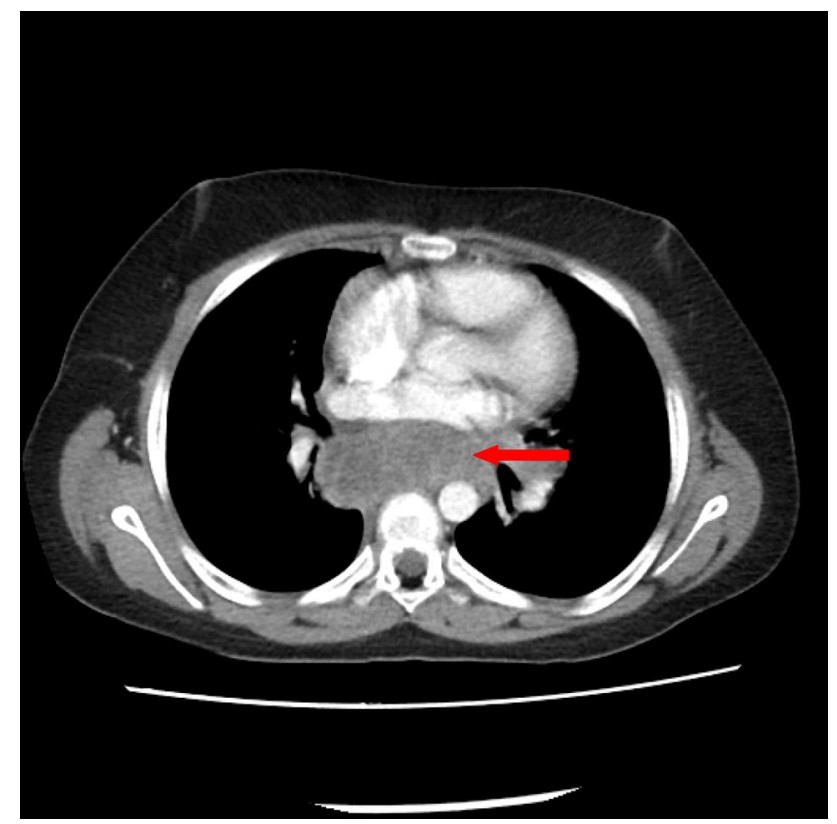

Fig. 3 Mediastinal multiple lymphadenopathies

serology were negative. Swelling lesion was resolved completely on the third week of therapy (Fig. 2).

Tuberculosis remains the major cause of morbidity and mortality by a single infectious agent, particularly in developing countries. Lymphadenitis is the most common extrapulmonary tuberculosis (TB) manifestation which, in developed countries, occurs more frequently in childhood [1]. Cases of mediastinal lymph node tuberculosis progression to the bronchi or esophagus due to the formation of fistulas have also been reported [2,3]. But skin fistules of mediastinal lymphadenitis in children are very rare (Fig. 3).

Conflict of interest On behalf of all authors, the corresponding author states that there is no conflict of interest.

\section{References}

1. Seddon JA, Shingadia D. Epidemiology and disease burden of tuberculosis in children: a global perspective. Infect Drug Resist. 2014;18(7):153-65.

2. Wigley FM, Murray HW, Mann RB, Saba GP, Kashima H, Mann JJ. Unusual manifestation of tuberculosis: TE fistula. Am J Med. 1976;60(2):310-4.

3. Ko Y, Lee HY, Lee YS, et al. Esophagomediastinal Fistula Secondary to Multidrug-resistant Tuberculous Mediastinal Lymphadenitis. Intern Med. 2014;53(16):1819-24. 